\title{
ARTIGOS
}

\section{La formación de maestros en la escuela normal superior de Cartagena de Indias de 2002 a 2010}

\author{
Marlén Rátiva Velandia \\ Universidad Pedagogica y Tecnológica de Colombia (UPTC) \\ Tunja, Boyacá, Colombia \\ ORCID https://orcide.org/0000-0002-7790-6199
}

\begin{abstract}
RESUMEN: Las Escuelas Normales Superiores en Colombia forman maestros en el programa de formación complementaria para desempeñarse en los grados transición y básica primaria. La formación debe tener énfasis en un área del conocimiento, desarrollar las capacidades de investigación pedagógica y de orientación y de acompañamiento pedagógico de los educandos por parte de los futuros maestros, entre otras demandas. Desde esta perspectiva, es importante conocer el origen, desarrollo y proyección de las Escuelas Normales Superiores, en el marco del contexto histórico y su incidencia en la formación de sujetos y colectivos sociales donde se reconoce al egresado normalista como líder crítico y transformador de realidades. El presente estudio se aborda desde la investigación histórica, acudiendo a los archivos de las Bibliotecas Luis Ángel Arango y Bartolomé Calvo, al Archivo General de la Nación, al de la Escuela Normal Superior de Cartagena de Indias y al Ministerio de Educación Nacional; para recolectar la información que acorde con Cardoso (1981) es necesaria para el desarrollo de la investigación, la cual se organiza, analiza e interpreta. Para dar cuenta de la formación de los futuros maestros se revisaron los planes de estudio de la escuela de 2002 a 2010 y una muestra de los proyectos de grado, que para ese entonces era requisito para obtener el título de maestro. Se determinaron como categorías de análisis la formación docente, la práctica pedagógica y la investigación.
\end{abstract}

PALABRAS CLAVE: Escuela Normal, formación docente, práctica pedagógica, investigación

\section{A formação de professores na escola normal superior de Cartagena de Indias de 2002 a 2010}

RESUMO: As escolas normais superiores da Colômbia formam professores para atuar nas séries do ensino fundamental e médio em um programa de treinamento complementar O treinamento deve enfatizar uma área do conhecimento, desenvolve as capacidades de pesquisa e orientação pedagógica e apoio pedagógico dos alunos pelos futuros professores, entre outras demandas. Nessa perspectiva, é importante conhecer a origem, o desenvolvimento e a projeção das Escolas Normais Superiores, dentro da estrutura do contexto histórico e seu impacto na formação de sujeitos e grupos sociais onde o normalista é reconhecido como líder crítico e transformador de realidades. O presente estudo é abordado desde a investigação histórica, passando pelos arquivos das bibliotecas Luis Ángel Arango e Bartolomé Calvo, pelo Arquivo Geral da Nação, pela Escola Normal Superior de Cartagena das Índias e pelo Ministério da Educação Nacional; Para 


\title{
autêntica
}

coletar as informações de acordo com Cardoso (1981), é necessário o desenvolvimento de uma pesquisa organizada, analisada e interpretada. Para dar conta da formação de futuros professores, foram revisados os currículos escolares de 2002 a 2010 e uma amostra dos projetos de graduação, que naquele momento era um requisito para obter o diploma de professor. A formação docente, a prática pedagógica e a pesquisa foram determinadas como categorias de análise

PALAVRAS-CHAVE: Escola Normal, formação docente, prática pedagógica, pesquisa.

\section{Teacher training at the superior normal School of Cartagena de Indias, 2002 - 2010}

\begin{abstract}
The Higher Normal Schools in Colombia train teachers in the complementary training program to perform in the preschool and primary. Training should have an emphasis on an area of knowledge, develop the capacities of pedagogical research and guidance and pedagogical support of students by future teachers, among other demands. From this perspective, it is important to know the origin, development and projection of the Higher Normal Schools, within the framework of the historical context and its impact on the formation of subjects and social groups where the normalist graduate is recognized as a critical leader and transformer of realities. The present study is approached from historical research, going to the archives of the Luis Ángel Arango and Bartolomé Calvo Libraries, to the General Archive of the Nation, to the Superior Normal School of Cartagena de Indias and to the Ministry of National Education; To collect the information that agrees with Cardoso (1981) is necessary for the development of research, which is organized, analyzed and interpreted. In order to account for the training of future teachers, the school curricula from 2002 to 2010 and a sample of the degree projects were reviewed, which at that time was a requirement to obtain the teacher's degree. Teaching training, pedagogical practice and research were determined as analysis categories.
\end{abstract}

KEYWORDS: Normal school, teacher training, pedagogical practice, research, 


\section{autêntica}

DOI https://doi.org/10.31639/rbpfp.v\%vi\%i.285

\section{INTRODUCCIÓN}

La formación docente en Colombia está a cargo de las Escuelas Normales Superiores y las Instituciones de Educación Superior. Las primeras, se rigen bajo políticas públicas educativas que determinan su organización y funcionamiento, igual que las IES pero en diferente condición, garantizando educación de calidad, pues esta;

(...) tiene una íntima relación con la acción del maestro, ya que es él quien propone y orienta las mediaciones con el conocimiento de los distintos saberes, con la formación ético social del ciudadano, con las posibilidades y los retos de la creatividad y la invención en todos los campos (AMAYA et al., 1997, p. 45).

De esta manera, el actuar del maestro ha estado determinado por la política pública y las decisiones institucionales; en algunos casos una vez emitidos los Decretos se realizan ajustes para implementarlos uno o dos años después con el fin de cumplir lo que allí se determina. Finalizando la década del noventa del siglo XX el Estado colombiano emitió el Decreto 3012 de 1997 y a finales de la primera década del siglo XXI el Decreto 4790 de 2008, los cuales reestructuraron las Escuelas Normales en Colombia, estableciendo la acreditación como una forma de garantizar la calidad y la continuidad en la formación de maestros para básica primaria en convenio con una Institución de Educación Superior, definiendo cuatro principios pedagógicos (educabilidad, enseñabilidad, contextos y pedagogía) para el diseño, el desarrollo de la propuesta curricular, el plan de estudios del programa de formación y la estructuración del ciclo en créditos académicos; enfocándose en el plan de estudios, la práctica pedagógica y la investigación en el ciclo complementario.

Entonces, las Escuelas Normales interesadas en continuar formando maestros acataron las disposiciones establecidas en los dos Decretos mencionados anteriormente, "dicho proceso se realizó en dos etapas: una de acreditación previa que duró 4 años (1998 a 2002) y otra denominada acreditación de calidad y desarrollo" (MEN, 2011, p. 1); lo cual permitió que 137 Escuelas Normales Superiores en 2010 adelantaran el proceso de verificación de las condiciones de calidad del programa de formación complementaria en modalidad presencial durante cinco años. A manera de ejemplo, se cita un caso, que permitirá conocer el origen, desarrollo y proyección de la Escuela Normal Superior de Cartagena de Indias en el marco del contexto histórico y su incidencia en la formación de maestros normalistas de su área de influencia. Para ello, el texto se estructura en tres apartados; trayecto histórico, formación de maestros, la práctica pedagógica y la investigación a la luz de los Decreto 3012 de 1997 y 4790 de 2008, para finalizar con las conclusiones.

En su totalidad, el texto da cuenta de la investigación histórica realizada a partir de la consulta de fuentes primarias como los periódicos del siglo XIX y XX que circulaban en Colombia cuya función era dar a conocer avances pedagógicos nacionales e internacionales, los informes de los Directores de Instrucción Pública, la implementación de la normatividad, entre otros, y las fuentes primarias y secundarias de la Escuela Normal Superior de Cartagena de Indias. Dicha información se encuentra localizada en los archivos de las Bibliotecas Luis Ángel Arango y Bartolomé Calvo, el Archivo General de la Nación, el Ministerio de Educación Nacional y la Escuela Normal.

\section{TRAYECTO HISTÓRICO DE LA ESCUELA NORMAL SUPERIOR DE CARTAGENA DE INDIAS}

En 1846 el gobernador Joaquín Posada Gutiérrez propuso fundar una Escuela Normal en Cartagena de Indias, fue así como el 15 de enero de 1847 mediante oficio número 19 solicitó la construcción de la Escuela Normal en el Convento La Merced; el primero de junio de 1847 se realizó la invitación para la construcción del salón 


\section{autêntica}

DOI https://doi.org/10.31639/rbpfp.v\%vi\%i.285

de la normal. Mediante el Decreto de Enero 17 de 1848, el gobernador Joaquín Posada Gutiérrez dio apertura a la Escuela Normal de la Provincia de Cartajena, manifestado en el artículo 1: “El día $1^{\circ}$ de Febrero próximo se abre la Escuela normal de la provincia i la primaria del distrito parroquial de la Catedral que le está anexa". (SEMANARIO DE LA PROVINCIA DE CARTAJENA, 1848, p. 1) Siendo su primer rector José Manuel Royo.

Con la llegada de la primera misión Alemana a Colombia, "contratada en 1872 bajo el gobierno del general Eustorgio Salgar para que organizara el sistema de las Escuelas Normales" (RÁTIVA, 2016, p. 171) se contrató desde Berlín a Julius Wallner como Director de la Escuela Normal de Cartagena de 1872 a 1877 porque era considerado por Salgar como:

(...) hombre honrado a carta cabal, conoce perfectamente su profesion de institutor pestalozziano, que ha practicado varios años en las Escuelas públicas de Prusia, teniendo de su habilidad pedagójica conocimientos científicos y conducta privada, los mejores certificados, así de la Escuela normal en que se educó. (GACETA DE BOLÍVAR, 1872, p. 31).

Sin embargo, solo empezó sus labores el 9 de enero de 1873, debido a que la Escuela no contó con una sede propia y adecuada; como lo expresó el artículo 10: "la Escuela normal i la primaria anexa se inaugurarán el día 9 de Enero". (GACETA DE BOLÍVAR, 1872, p. 198). En 1874 la Escuela Normal de Bolívar concedió el primer diploma de maestro de Escuela Superior a Vicente Tapia (LA ESCUELA NORMAL (LEN), 1874, p. 1) expedido en Cartagena el 6 de julio. Para esa época la formación de los maestros se caracterizó por estar organizada en lecciones semanales de lectura para conseguir habilidad práctica al leer del molde y manuscritos, procurando buena articulación y pronunciación; de escritura en lo referido a caligrafía y ortografía; de aritmética para aprender las operaciones básicas y conteo; de enseñanza objetiva referida a conversaciones sobre los objetos y su estado, sustantivos, adjetivos, verbos, el hombre en sociedad; de historia sagrada caracterizada por contar las historias del Antiguo Testamento y de Gimnasia en las pausas.

En los años de 1876 y 1877 las Escuelas Normales fueron cerradas debido a otra guerra civil, "la Escuela Normal, la cual estuvo clausurada por orden del poder ejecutivo nacional por más de un año y medio. Varios alumnos cambiaron entonces el libro por el fusil y lucharon en los campos de Piaurichon por las libertades públicas". (MENESES, 2012, p. 168). Al inicio del año 1878 los alumnos volvieron a las aulas de la Escuela Normal de Varones de Bolívar, se concedieron los diplomas de capacidad para el desempeño de las funciones de Maestros de Escuela Superior a: "José María P. Urueta, Alejandro Vásquez P., José E. Nova i José Vicente García". (LEN, 1878, p. 1).

Una vez finalizado el contrato de Wallner, fue nombrado rector de la Escuela Normal Antonio Cátulo Royo mediante Decreto 143 del 18 de Mayo de 1878 (DIARIO DE BOLÍVAR, 1878, p. 1186), y en el mes de septiembre del mismo año, se abrió la Escuela Normal de Institutoras de Bolívar en la ciudad de Cartagena con una Escuela Anexa; Eugenia Moré fue nombrada directora y Zoila Moré como subdirectora. En enero de 1879 la Escuela Normal de Varones se trasladó a un nuevo local, dejando de compartir el espacio con el Colegio del Estado, una vez se obtuvo la autorización por parte de la Secretaría de lo interior i de relaciones exteriores. Ese mismo año el 2 de marzo, se concedieron diplomas de capacidad para el desempeño de maestro en una escuela superior a Antonio Gutiérrez con la calificación de aprobado con plenitud, Carlos Espinosa con la calificación de notable y José Manuel del Castillo con calificación sobresaliente (LEN, 1876, p.1). Hay que mencionar, además que la Escuela Normal funcionó con cierta normalidad hasta 1884; tiempo en el cual vuelve a cerrarse debido a los conflictos armados y el poco dinero para su sostenimiento. 


\section{autêntica}

DOI https://doi.org/10.31639/rbpfp.v\%vi\%i.285

La Escuela vuelve a funcionar en 1886, pero en 1895 sufre otro cierre como producto de la guerra civil debido a la utilización de "espacios de educación como "servicios de cuartel", tal como sucedió con la Escuela Normal Superior de Cartagena, clausurada y convertida en centro de operaciones militares" (ACEVEDO, 2009, p. 114).

En 1897 vuelve a funcionar, para ese entonces, la Escuela Normal de Institutores tenía como director a Pedro Araujo Blanco, como subdirector a Eusebio Celio Fernández y como director de la anexa Miguel García Fernández quienes convocaron a exámenes los días 15, 17, 19 y 21 de noviembre. De primer año se evaluarían ocho alumnos, de segundo año cinco, uno de cuarto año y de la escuela anexa ciento tres alumnos. Pero, en 1899 vuelve a cerrarse debido a la guerra de los mil días (RÁTIVA, 2017b, p. 182).

Entre 1906 y 1914 los directores de Instrucción Pública realizaron varias visitas a la Escuela Normal Nacional de Institutoras del Departamento para reportar lo que allí se hacía en relación a la práctica pedagógica, la asistencia de las futuras maestras y las actividades que desarrollaban. Para el año de 1932 se cierran las siguientes Escuelas Normales: "Escuela Normal Central de Institutoras de Bogotá y las Escuelas Normales de Institutores de Barranquilla, Bucaramanga, Cartagena, Cúcuta, Ibagué, Manizales, Medellín y Neiva" (VALENCIA, 2006, p. 237), lo cual indica que la Escuela Normal de Señoritas continuó formando maestras en el departamento de Bolívar e incluso determinando que la docencia sería exclusiva y predominantemente femenina. Para el año de 1939 las Escuelas Normales incluyeron en tercer año del plan de estudios psicología y metodología, las cuales no habían sido tenidas en cuenta en años y planes anteriores, luego se sumaron metodología de la enseñanza, y pedagogía y metodología.

A partir del Decreto número 12 de 1942 se reorganizó la Escuela Normal de Señoritas de Cartagena y la Escuela anexa como requisito para reconocer los estudios y expedir los diplomas de maestras a las alumnas que se graduaran, se identificó que debido al pequeño espacio del local de la Escuela Normal solamente se contaría con cinco grupos uno por cada año de estudio.

En 1978 el Ministerio de Educación Nacional evaluó todas las Escuelas Normales teniendo en cuenta indicadores de calidad. Las Escuelas Normales fueron ubicadas en cinco categorías; la primera era categoría de calidad superior y la quinta calidad inferior; aquellas que estuvieran en esta última, debían cambiar de modalidad; tal fue el caso de la Escuela Normal Piloto de Bolívar Nuestra Señora del Carmen y tuvo que someterse a la transformación progresiva, pasó a ser Concentración Educativa Alberto Elías Fernández Baena (bachillerato pedagógico), fundada el 17 de marzo de 1982. Transcurridos trece años, con el Decreto 1135 del 13 de Octubre de 1995 se volvió a dar vida a la Escuela Normal para continuar con la formación de docentes para la Educación Básica Primaria en la capital del departamento de Bolívar y el Ministerio de Educación Nacional aprobó su reestructuración mediante Resolución No. 3081 de 1995.

En el año 2000 se inició el proceso de reestructuración en convenio con la Universidad del Atlántico, en septiembre alcanzó la acreditación previa otorgada por el Ministerio de Educación Nacional mediante Resolución No 2564 del 29 de Septiembre de 2000, en 2001 empezó a desarrollar la propuesta del Ciclo Complementario a partir de los campos de formación: saber pedagógico, saber disciplinar y comunicación, significación y tradición cultural, en 2002 fue reconocida oficialmente como Escuela Normal Superior de Cartagena de Indias y según Resolución 4093 de Noviembre de 2004 recibió la Acreditación de Calidad y Desarrollo, actualmente funciona bajo la dirección del profesor Álvaro Hernández Castellón. 


\section{autêntica}

DOI https://doi.org/10.31639/rbpfp.v\%vi\%i.285

\section{LA FORMACIÓN DE MAESTROS EN LA ESCUELA NORMAL SUPERIOR DE CARTAGENA DE INDIAS SEGÚN LOS DECRETO 3012 DE 1997 Y 4790 DE 2008}

La última década del siglo XX tuvo cambios significativos para la educación colombiana, iniciando con una nueva Constitución Política Nacional en 1991, en la cual la educación se estableció como un derecho de la persona y un servicio público, propuso que la enseñanza estaría a cargo de personas de reconocida idoneidad ética y pedagógica. Tres años después, con la Ley General de Educación 115 de 1994, se propuso una nueva concepción del maestro como constructor, diseñador e investigador de los procesos educativos; proyectando una visión de la educación como un proceso de formación permanente, personal, cultural y social fundamentada en una concepción integral de la persona humana, de su dignidad, de sus derechos y de sus deberes. En relación con la formación docente, se propuso que fuera de alta calidad científica y ética, que la teoría y la práctica fueran la parte fundamental del saber del educador y que se fortaleciera la investigación pedagógica y disciplinar. Confirmó el rol de las Escuelas Normales como formadoras de docentes para la educación inicial, llamándolas Unidades de Apoyo Académico, cumpliendo el requisito de estar debidamente reestructuradas, aprobadas por el Ministerio de Educación Nacional y en convenio con una Institución de Educación Superior para ofrecer la formación complementaria con un énfasis, el cual se esperaba fuera definido teniendo en cuenta el contexto institucional. Además de estar debidamente acreditadas en forma previa según las disposiciones del Ministerio de Educación.

Para hacer tangible esta propuesta en 1997 se emitió el Decreto 3012 el cual definió la pedagogía como disciplina fundante, se asumió al docente como un investigador, planteó condiciones para que las Escuelas Normales en proceso de reestructuración pudieran ser aprobadas como Escuela Normal Superior y operar como unidades de apoyo académico a la educación inicial de los profesores, estableciendo los grados 12 y 13 como ciclos complementarios, exigió un comité interinstitucional integrado por un representante de la Universidad con la que se tenía convenio y un representante de la Junta de Educación Distrital o Municipal para evaluar dicho convenio. Además de esto, destacó la importancia de incluir las tecnologías como parte esencial del currículo y estableció como núcleos del saber el uso pedagógico de los medios de comunicación y el manejo de una segunda lengua.

Una década después se promulgó el Decreto 4790 de 2008 el cual complementó al anterior en lo referido a las condiciones de calidad del ciclo complementario bajo cuatro principios pedagógicos: educabilidad, enseñabilidad, contextos y pedagogía, y condiciones de calidad como: las prácticas docentes en la formación complementaria, el plan de estudios y la práctica pedagógica relacionada con los temas de preescolar y básica primaria, las innovaciones en el campo educativo que fomenten la investigación y la estructuración de los planes de estudio en créditos académicos; como dice Figueroa (2015) las Escuelas iniciaron su proceso de autoevaluación para analizar las condiciones de calidad descritas anteriormente.

A su vez, la propuesta del Ciclo Complementario se organizó a partir de tres dimensiones: identidad del maestro, conocimiento y comunicación, significación y cultura, estos a su vez se agruparon en los campos de formación: saber pedagógico y competencias del maestro (identidad del maestro), saberes disciplinares (conocimiento), y comunicación, significación y tradiciones culturales (comunicación, significación y cultura). Para abordar esta propuesta se determinaron como acciones pedagógicas los proyectos de investigación de aula y las clases integradas.

El Decreto 3012 estableció que las Escuelas Normales Superiores debían diseñar y ejecutar propuestas de investigación pedagógica y planes de asistencia técnico-pedagógica para las Instituciones Educativas de 


\title{
autêntica
}

DOI https://doi.org/10.31639/rbpfp.v\%vi\%i.285

su área de influencia; con el fin de atender a este requisito en la Escuela Normal Superior de Cartagena de Indias se propuso la línea investigativa: formación de formadores; desde lo pedagógico, lo disciplinar y el rescate de tradiciones culturales y dentro del proyecto de práctica pedagógica se desarrolló la asistencia en las escuelas con las cuales se firmó el convenio para orientarlas, asesorarlas y acompañarlas en el desarrollo institucional y fortalecimiento del Proyecto Educativo Institucional.

\section{LA PRÁCTICA PEDAGÓGICA Y LA INVESTIGACIÓN EN LA ESCUELA NORMAL SUPERIOR DE CARTAGENA DE INDIAS}

La práctica pedagógica es entendida como

\begin{abstract}
un proceso formativo en el cual se espera que el futuro maestro adquiera saberes que le permitan a través de la experimentación, diseñar y ejecutar actividades que influyan en su proceso de enseñanza - aprendizaje para ser maestro y en el de sus estudiantes; porque es a través de la experiencia como se aprende, se aprende a ser maestro. Por consiguiente, tiene dos dimensiones, la primera corresponde a la formativa y la segunda a la experiencia; en la primera confluyen la normatividad y las decisiones institucionales, y en la segunda la propuesta investigativa y pedagógica, y los procedimientos. (RÁTIVA, 2017a, p.3).
\end{abstract}

Acorde con Zuluaga (1995) el campo de experimentación permite legitimar la investigación y la docencia, y confrontar los procesos de apropiación de conocimientos y la producción de saber en la realidad porque estos deben ser transformadores del quehacer del docente. Desde esta perspectiva, los ajustes realizados como producto de la exigencia de la normatividad se reflejaron en la práctica pedagógica iniciando en la dimensión formativa; los futuros maestros profundizaron en lenguaje, asumiéndolo como el eje transversal del conocimiento, el cual facilita la interdisciplinariedad y se aborda en todas las áreas del conocimiento; al incorporar la investigación para la realización de un proyecto de investigación.

Dichos cambios empezaron en 2001, el maestro normalista en el ciclo complementario realizaba la práctica pedagógica en las Instituciones Educativas con las que se firmó el convenio, iniciando con la observación desde el primer semestre para que al final de éste se presentara la propuesta investigativa a desarrollar, durante ese tiempo se tomaban registros de la clase para hacer la reconstrucción pedagógica del contexto; se describían situaciones de convivencia, metodología del profesor titular en diferentes clases, interacción profesor - estudiantes y estudiantes - estudiantes, actividades recurrentes, las actividades propuestas por los maestros en formación que hacían parte de las proyectos de grado, etc.

En los siguientes semestres, una vez establecida la propuesta investigativa se fundamentaba teóricamente, se definía la intervención pedagógica diseñando actividades y ejecutándolas en el aula para recolectar la información que luego era analizada, interpretada y validada; durante ese tiempo continuaban los registros. Los proyectos de investigación se enfocaron en resolver problemáticas relacionadas con la lectura, la escritura, las competencias en lenguaje, la adquisición del código escrito, la comprensión de textos, el aprendizaje, la memoria colectiva, la convivencia, el uso de material didáctico, el aprendizaje de la matemática, la argumentación, la tradición oral, entre otros, los cuales se inscriben en las tres dimensiones (identidad del maestro, conocimiento y comunicación, significación y cultura) y evidencian la interdisciplinariedad.

En este proceso el maestro en formación activaba e integraba los conocimientos adquiridos en el ciclo, referidos a los procesos investigativos y a los aportes brindados en los campos, para establecer su propuesta 


\section{autêntica}

DOI https://doi.org/10.31639/rbpfp.v\%vi\%i.285

investigativa que atendía desde lo investigativo a resolver el problema identificado y desde lo pedagógico a integrar métodos de enseñanza, didáctica y el saber disciplinar. Es así como parafraseando a Herbart (1935):

la educación es el cuidado de aptitudes que permiten la evolución, la adquisición de ideas, la modelación de los deseos y la voluntad para llegar a ser autónomo, libre, capaz de gobernarse a sí mismo; esto se evidencia en los 120 proyectos desarrollados por los maestros en formación y que les permitieron obtener el título de Normalista Superior (RÁTIVA, 2017b, p. 249).

Para los maestros y directivos de la Escuela Normal Superior de Cartagena de Indias "los proyectos se relacionan con la realidad social y sus tradiciones, la labor educativa es contextualizada y trata de darle respuesta a las necesidades e intereses de las comunidades" (ENSCI, 2010, sp). Tanto la práctica pedagógica como la investigación es un proceso que se desarrolló por etapas integrando la teoría y la práctica, la primera es la sensibilización; los maestros en formación tienen su primer contacto con la población estudiantil por periodos cortos de tiempo desde grado noveno para realizar observación, esto da paso a la segunda etapa: la adaptación que progresivamente desde noveno se empieza a familiarizar con la profesión y en los grados décimo y once han comprendido en qué consiste la labor docente, sus implicaciones y su importancia, como producto de esto se toma la decisión de continuar su formación en el ciclo complementario, en el cual se llevan a cabo las etapas de fundamentación y profundización, caracterizadas por continuar la formación del futuro maestro en aspectos disciplinares, investigativos y didácticos.

\section{GRÁFICA NO. 1. ETAPAS DE LA PRÁCTICA PEDAGÓGICA Y LA INVESTIGACIÓN}

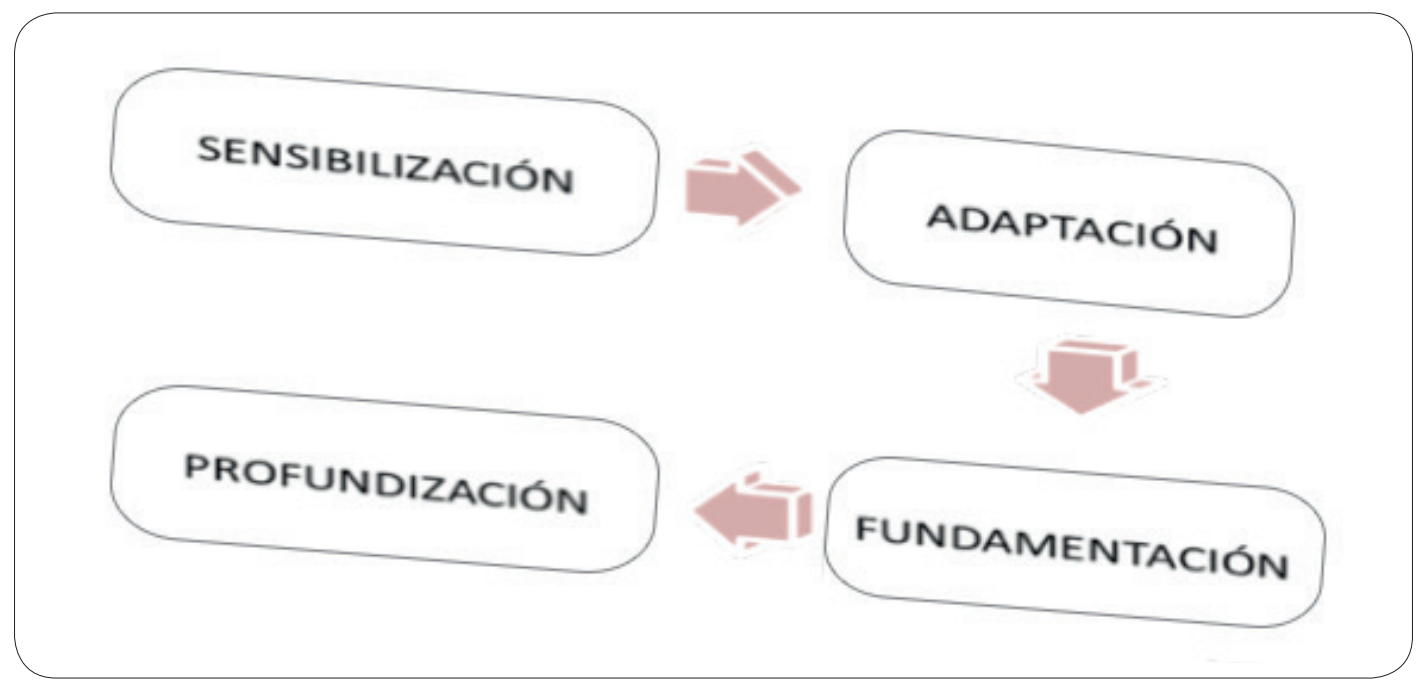

*Fuente: Elaboración propia

De lo anterior, se puede afirmar que la práctica pedagógica en la dimensión de experiencia se desarrolló desde la observación, el descubrimiento de intereses y necesidades, el análisis, la planificación, la fundamentación teórica, la reflexión y la transformación, para lo cual los maestros en formación asumieron desde una perspectiva diferente a la tradicional la enseñanza-aprendizaje; en esta oportunidad con un alto contenido investigativo que problematiza el aula desde la observación y de la lectura del contexto, transformándola en una práctica pedagógica investigativa y que según de Zuluaga (1995) en el proceso de reestructuración de las Escuelas Normales se debe evitar hablar de dos polos; uno que corresponde a lo que se enseña y otro a 


\section{autêntica}

DOI https://doi.org/10.31639/rbpfp.v\%vi\%i.285

lo que se investiga, es necesario que las Escuelas se ubiquen en una línea horizontal en la que estudiantes y maestros investiguen, que conformen semilleros de investigación, que escriban y que circulen el conocimiento.

A partir del Decreto 4790 de 2008, desde el año 2010 la Escuela Normal Superior de Cartagena de Indias reestructuró la propuesta investigativa eliminando los proyectos de grado por grupos de investigación liderados por maestros de la Escuela Normal, en estos espacios los maestros en formación se inscriben en un proyecto, realizan las actividades propuestas por el investigador principal y las que se determinaron en el proyecto pedagógico de aula, se documentan, analizan, sistematizan y los productos de la investigación se publican en la revista de la Escuela o en otros medios, este trabajo les permite obtener el título de maestros. Sin embargo, es necesario que se revise cómo se está abordando la investigación, la formación del maestro y la práctica pedagógica para evitar lo que Zuluaga denomina como los dos polos; es decir que en el proceso de la autoevaluación la mirada hacia la formación de los maestros en el ciclo complementario debe estar enfocada en precisar los elementos que caracterizan la formación de los maestros. 


\section{autêntica}

DOI https://doi.org/10.31639/rbpfp.v\%vi\%i.285

\section{CONSIDERACIONES FINALES}

Construir el trayecto histórico, institucional y pedagógico de las Escuelas Normales Superiores contribuye a conocer sus orígenes, su desarrollo, su incidencia en la formación de sujetos y su proyección; lo cual permite que desde el ámbito académico se determinen lazos que ayuden a seguir fortaleciéndolas, empoderarlas y posicionarlas socialmente como las pioneras en la formación de maestros que aún tienen mucho que aportar en nuestro país.

Desde 1978 las Escuelas Normales en Colombia han estado en proceso de evaluación, se puede afirmar que con la evaluación a las Escuelas realizada por el Ministerio de Educación Nacional en 1978, se llevó a cabo una primera acreditación, de allí que el Estado decidió cerrar escuelas normales por no cumplir con las condiciones de calidad educativa que para esa época se determinaron (eficiencia, recursos humanos y dotación física de los planteles) y que en la actualidad están definidas y se exigen según lo estipulado en el actual Decreto que rige a las Escuelas, Decreto 4790 de 2008.

Se identifica que la formación de los maestros ha estado permeada por las decisiones institucionales, de política pública educativa; para este caso la emitida durante los dos periodos de gobierno del entonces presidente Álvaro Uribe Vélez, tiempo en el cual los factores económicos de orden mundial; dieron origen a los Decretos 3012 de 1997 y 4790 de 2008 y que reestructuraron las Escuelas Normales Superiores en Colombia sumergiéndolas en la dinámica de eficiencia, efectividad, pertinencia y competitividad; siendo éstas exigencias a nivel internacional que también deben cumplir las Instituciones de Educación Superior.

A su vez, las políticas públicas educativas están presentes en la vida institucional de la Escuela Normal Superior de Cartagena de Indias, desde su inicio, pasando por los cierres y reaperturas, en su progreso y en la actualidad. Es desde ella que la Escuela recibió orientaciones para ser reestructurada, renovada y transformada, para proponer un plan de estudios estructurado desde la investigación, para establecer convenios que ayudaron a su acreditación y a la continuidad de los estudios universitarios para sus egresados, para pensar en que su función como formadora de formadores debía cumplir con unos criterios de calidad y de exigencia académica necesarios para brindar una educación más equitativa, justa y pertinente para su zona de influencia.

Indiscutiblemente, la Escuela Normal Superior de Cartagena de Indias tenía claro que su función era la de formar maestros y que para esto era necesario proponer una línea de investigación desde la cual se reflexionara en torno a la formación, la profesión, las necesidades de los estudiantes, la vida institucional y como ellos lo mencionan el rescate de las tradiciones culturales; de esta manera desde la línea formación de formadores, se inscribe no solamente a cumplir su misión de formadora de formadores sino a acatar la normatividad como un acto testimonial de su compromiso, interés y deseo de contribuir con la educación del Distrito de Cartagena de Indias y del departamento de Bolívar.

La Escuela Normal Superior de Cartagena de Indias recurre a las prácticas investigativas para fortalecer en los maestros en formación, actitudes, habilidades y competencias para la construcción de saberes pedagógicos y disciplinares a través del trabajo en el aula, la observación en la búsqueda de soluciones a problemas de la realidad, del contexto y del entorno de la Escuela. Esto hace que desde lo conceptual se adopte la formación a partir de la investigación, con prácticas reflexivas que posibiliten la transformación de las prácticas pedagógicas y la concepción de educación, con maestros que indaguen constantemente su contexto para plantear alternativas de solución que favorezcan los procesos de enseñanza - aprendizaje y su propia formación. 


\section{autêntica}

DOI https://doi.org/10.31639/rbpfp.v\%vi\%i.285

\section{REFERENCIAS}

ACEVEDO, R. Escuelas y políticas educativas en la provincia de Cartagena entre 1903 y 1919. El taller de la historia, v. 1, n. 1. 2009.

ALARCÓN, L. Maestros y escuelas normales en el caribe colombiano durante el Régimen Federal. In Revista Historia de la Educación Latinoamericana, v. 14, n. 18, p. 155-182, 2012.

AMAYA, G. [et al]. La formación de los educadores en Colombia. Bogotá: Instituto para la Investigación Educativa y el Desarrollo Pedagógico, 1997.

ARCHIVO GENERAL DE LA NACIÓN. Diario de Bolívar. no. 1906 (Cartajena: 15 de Junio de 1878), 1186.

BIBLIOTECA BARTOLOMÉ CALVO. Semanario de la Provincia de Cartajena. no. 289, microfilm 304 (23 de Enero de 1848), 1.

BIBLIOTECA BARTOLOMÉ CALVO. Gaceta de Bolívar. no. 800, microfilm 781 (Cartajena: 25 de Febrero de 1872), 31.

BIBLIOTECA BARTOLOMÉ CALVO. Gaceta de Bolívar. no. 840, microfilm 781 (Cartajena: 31 de Diciembre de 1872), 198.

BIBLIOTECA LUIS ÁNGEL ARANGO. La Escuela Normal. Periódico Oficial de Instrucción Pública. Tomo V, no. 160. (Bogotá: 24 de Enero 1874), 1.

BIBLIOTECA LUIS ÁNGEL ARANGO. La Escuela Normal. Periódico Oficial de Instrucción Pública. Tomo VII, no. 293. (Bogotá: 19 de Diciembre, 1878), 1.

BIBLIOTECA LUIS ÁNGEL ARANGO. La Escuela Normal. Periódico Oficial de Instrucción Pública. Tomo VII, no. 264. (Bogotá: 29 de Abril, 1876), 1.

CARDOSO, Ciro. Introducción al trabajo de la investigación histórica. Conocimiento, método e historia. Barcelona: Crítica, 1981.

CARTAGENA, Secretaría de Educación Distrital de Cartagena. Decreto 1135. Por el cual se da reapertura a la Escuela Normal de Cartagena de Indias. (13 Octubre 1995).

COLOMBIA, Ministerio de Educación Nacional. Evaluación de las Escuelas Normales de Colombia, 1-3, (1978).

COLOMBIA, Ministerio de Educación Nacional. Ley 115. Por la cual se expide la Ley General de Educación. (8 Febrero 1994).

COLOMBIA, Ministerio de Educación Nacional. Resolución 3081. Por la cual se aprueba la reestructuración de la Escuela Normal Nuestra Señora del Carmen como Escuela Normal Superior. (1995).

COLOMBIA, Ministerio de Educación Nacional. Decreto 3012. Por el cual se adoptan disposiciones para la organización y el funcionamiento de las Escuelas Normales Superiores. (19 Diciembre 1997). 


\section{autêntica}

DOI https://doi.org/10.31639/rbpfp.v\%vi\%i.285

COLOMBIA, Ministerio de Educación Nacional. Resolución 2564. Por el cual se concede la acreditación previa. (29 Septiembre 2000).

COLOMBIA, Ministerio de Educación Nacional. Resolución 4093. Por la cual se concede la Acreditación de Calidad y Desarrollo a la Escuela Normal Superior de Cartagena de Indias. (10 Noviembre 2004).

COLOMBIA, Ministerio de Educación Nacional. Decreto 4790. Por el cual se establecen las condiciones básicas de calidad del programa de formación complementaria de las Escuelas Normales Superiores y se dictan otras disposiciones. (19 Diciembre 2008).

COLOMBIA, Constitución Política de Colombia. (2011). Bogotá: Espiral ediciones.

COLOMBIA, Ministerio de Educación Nacional. Escuelas Normales Superiores acreditadas. (2011). Recuperado el 8 de mayo de 2015 de http://www.mineducacion.gov.co/1621/articles-208800_archivo_pdf_ens_acreditadas_junio2_2011.pdf

ESCUELA NORMAL SUPERIOR DE CARTAGENA DE INDIAS. Documento para Acreditación de Alta Calidad. (2010) (sp).

FIGUEROA, C. De la tradición histórica de las Escuelas Normales Superiores a la visibilización institucional. Un aporte a la formación de educadores en el marco de las políticas públicas en Colombia durante los siglos XX y XXI. In: MARTÍNEZ, Armando; PASCUAL, José y GUZMÁN, Juan (Comps.). Evidencias, conjeturas y hallazgos en torno a la Historia de la Educación Latinoamericana. México: Amaya Ediciones, 2015, p. 1088-1106.

HERBART, Johann. Pedagogía General. Derivada del fin de la educación. Madrid: Espasa Calpe S.A., 1935.

RÁTIVA, M. Las Escuelas Normales en Suramérica "El normalismo en vía de extinción" Colombia ¿cómo estamos? In Revistas Hojas y Hablas, n. 13, p. 169-178. 2016. Disponível em http://revistas. unimonserrate. edu.co:8080/hojasyhablas/issue/view/6

RÁTIVA, M. La práctica pedagógica en la Escuela Normal Nacional de Institutoras de Cartagena de 1900 a 1930. In. III Coloquio Nacional y I Internacional de estudiantes de doctorado en educación. Universidad Santo Tomás, Bogotá, (junio, 2017a).

RÁTIVA, Marlén. La Escuela Normal Superior de Cartagena de Indias, una historia en construcción. Mirada a la práctica pedagógica de 1998 a 2010. Tesis (Doctorado en Educación) Universidad Pedagógica y Tecnológica de Colombia - Tunja, 2017b.

VALENCIA, Carlos. Las Escuelas Normales y la formación del magisterio. Primera Mitad del Siglo XX. Manizales: Universidad de Caldas, 2006.

ZULUAGA, O. Investigación y experiencia en las Escuelas Normales. In Revista Educación y Pedagogía, v. 08, n. 16, p. 54-162, 1996. Disponible en http://aprendeenlinea.udea.edu.co/revistas/index.php/revistaeyp/ article/view/5713/5133 


\section{autêntica}

DOI https://doi.org/10.31639/rbpfp.v\%vi\%i.285

VALENDIA, M. R.;

La formación de maestros en la escuela normal superior de Cartagena de Indias de 2002 a 2010

Formação Docente - Revista Brasileira de Pesquisa sobre Formação de Professores

Vol. 11, no. 22 (p. 111-124) 31 dez. 2019 
